\title{
Experimental and Numerical Investigations of Three-Dimensional Flows around Propellers in Low-Reynolds Number Flows
}

\author{
By Koichi YonEZAwA ${ }^{1)}$, Shigeru SunADA ${ }^{2)}$, Yudai Goto ${ }^{1)}$, Takashi HAYASHIDA ${ }^{2)}$, \\ Masayuki ANYOJI ${ }^{3)}$ and Yoshinobu TSUJIMOTO ${ }^{1)}$ \\ 1) Graduate School of Engineering Science, Osaka University, Toyonaka, Japan \\ ${ }^{2)}$ Graduate School of Engineering, Osaka Prefecture University, Sakai, Japan \\ 3) Institute of Space and Astronautical Science, Japan Aerospace Exploration Agency, Sagamihara, Japan
}

(Received June 24th, 2013)

\begin{abstract}
A propeller for small scale unmanned aerial vehicle (UAV) is used in low-Reynolds number conditions. In order to obtain high thrust and efficiency under low-Reynolds number conditions, the blade aspect ratio becomes small. The flow around the propeller with low-aspect ratio becomes quite three-dimensional. In the present study, experimental and numerical investigations are carried out to clarify effects of the three-dimensional flow on the propeller performance focusing on vortices associating with the low-Reynolds number flows.
\end{abstract}

Key Words: Propeller, Low-Aspect Ratio, Low-Reynolds Number, Leading Edge Vortex, Tip Vortex

\section{Nomenclature}

$$
\begin{aligned}
& c \text { : Blade chord length } \\
& C_{T} \quad: \text { Thrust coefficient }\left(=\frac{\text { Thrust }}{1 / 2 \rho U_{a}^{2} \pi R^{2}}\right) \\
& C_{Q} \quad: \quad \text { Torque coefficient }\left(=\frac{\text { Torque }}{1 / 2 \rho U_{a}^{2} \pi R^{3}}\right) \\
& r \quad: \text { Radius } \\
& R \quad: \text { Propeller radius } \\
& \operatorname{Re} \quad: \quad \text { Reynolds number }\left(=\frac{\rho c \sqrt{U_{a}^{2}+(3 / 4 R \omega)^{2}}}{\mu}\right) \\
& u^{\prime} \quad \text { : Axial component of induced velocity } \\
& U \text { : Axial velocity in stationary flame } \\
& U_{a}: \text { Forward velocity } \\
& v \quad \text { : Circumferential component of relative velocity } \\
& v^{\prime} \text { : Circumferential component of induced velocity } \\
& V \quad \text { : Circumferential velocity in stationary flame } \\
& \beta \quad \text { : Blade twist angle } \\
& \eta \quad: \text { Propeller efficiency }\left(\eta=\frac{\text { Thrust } \cdot U_{a}}{\text { Torque } \cdot \omega}\right) \\
& \omega \text { : Propeller angular velocity }
\end{aligned}
$$

\section{Introduction}

Propellers for small scale unmanned aerial vehicle (UAV) are operated under Reynolds numbers lower than $10^{4}$. In the low-Reynolds number flow, aerodynamic characteristics of propeller blade become worse. Therefore, it can be beneficial to adopt blades with the low-aspect ratio and with the large blade chord length in order to increase the Reynolds number.

Various optimum design methods are developed for the propeller design. In the present study, an optimum blade design method is adopted to design the test propeller, which is proposed by Adkins and Liebeck ${ }^{1)}$. In this method, propeller induced velocity is calculated based on a blade element theory using lift and drag force data of airfoil, and finally, radial distributions of the blade twist angle and the chord length are obtained. Although validities of Adkins-Liebeck method has been confirmed for high aspect ratio blade designs at Reynolds numbers higher than $10^{5}$, applications for low-aspect ratio blade at Reynolds numbers lower than $10^{4}$ have not been discussed enough.

One of typical examination of low-Reynolds number flow around rotating low-aspect ratio blade is rotary seeds of maple tree $^{2,3)}$. Many studies about flow patterns around the seed have been carried out to clarify how to increase the flight distance. It has been clarified that a leading edge vortex (LEV) plays an important roll to increase the lift force ${ }^{4-6)}$ Additionally, the LEV has spanwise velocity component due to low-aspect ratio of the airfoil and the spanwise velocity component enhance the stability of the LEV.

The present study aims to clarify three-dimensional flow characteristics around the propeller blade due to low-aspect ratio and due to low-Reynolds number in order to develop a propeller with the high efficiency. For this purpose, experiments and numerical simulations are carried out. In addition to the high propeller efficiency, it is also important to increase the thrust in order to minimize the propeller diameter. The blade skew with a backward sweep angle is also examined.

The triangle airfoil ${ }^{7)}$ is adopted as the blade cross section of the test propeller as shown in Fig. 1 (a). The triangle airfoil has the large lift-drag ratio in flows with the order of Reynolds number of $10^{3}$. The radial distributions of the blade twist angle and the chord length are determined using Adkins-Liebeck method. Two kinds of planform are examined as shown in Fig. 1 (b) and (c). The one is termed as the original blade and the 


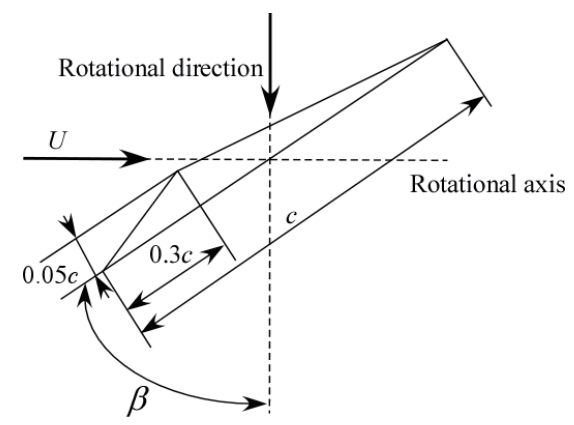

(a) Blade cross section

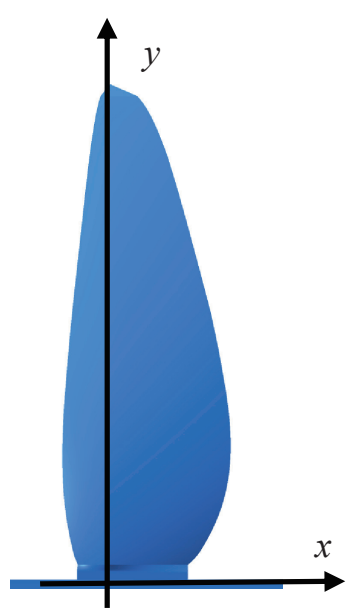

(b) Original blade

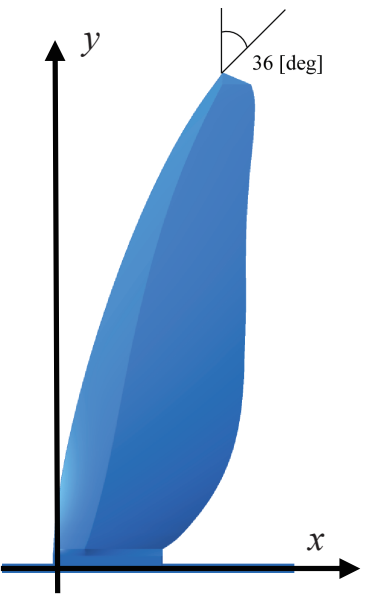

(c) Skewed blade

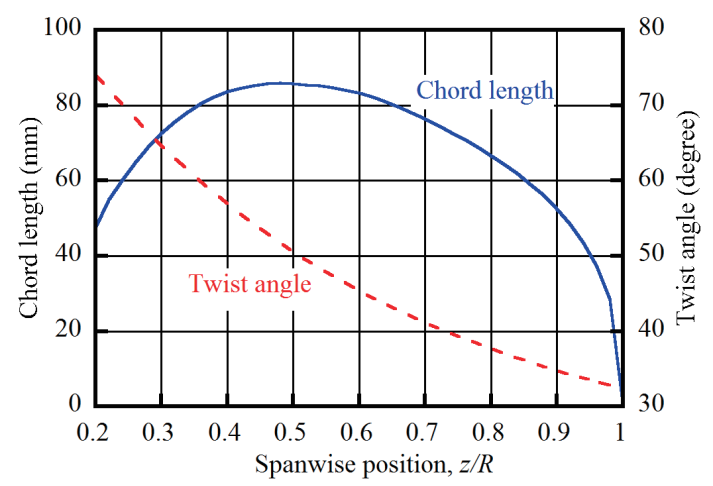

(d) Distributions of chord length ( $\mathrm{mm}$ ) and blade twist angle (deg) along blade span.

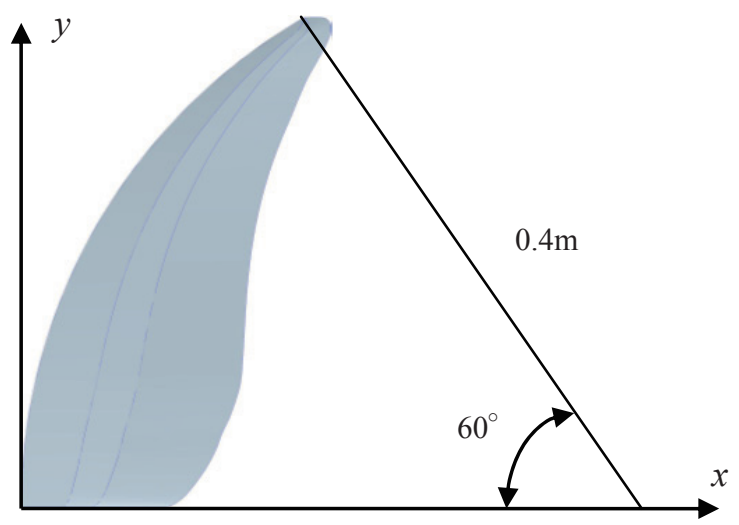

(e) Outline of leading edge of the skewed blade when the variation of $\beta$ from its value at the root is ignored

Fig. 1. Blade shapes of test propellers.

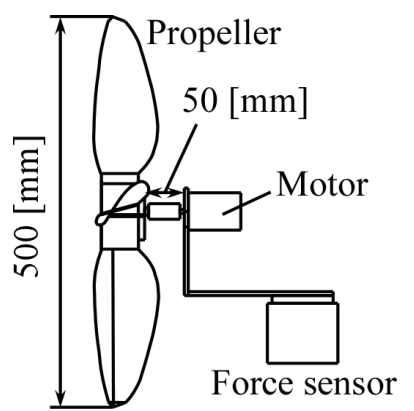

(a) Thrust measurement configuration

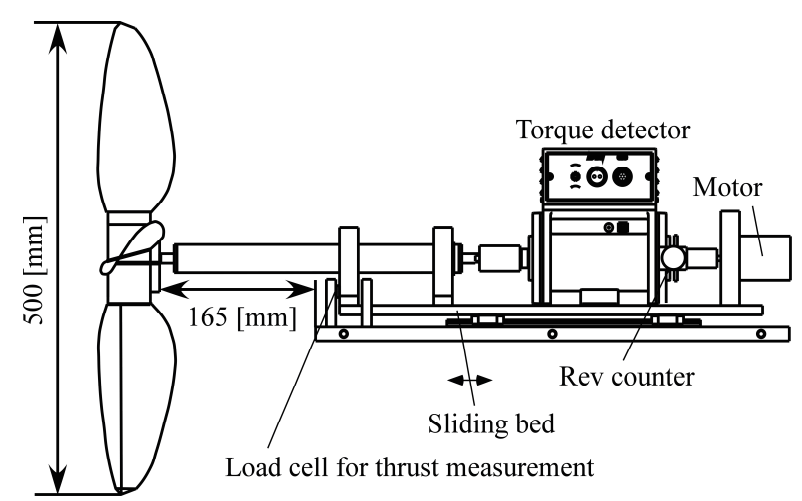

(b) Thrust and torque measurement configuration

Fig. 2. Propeller test configurations.

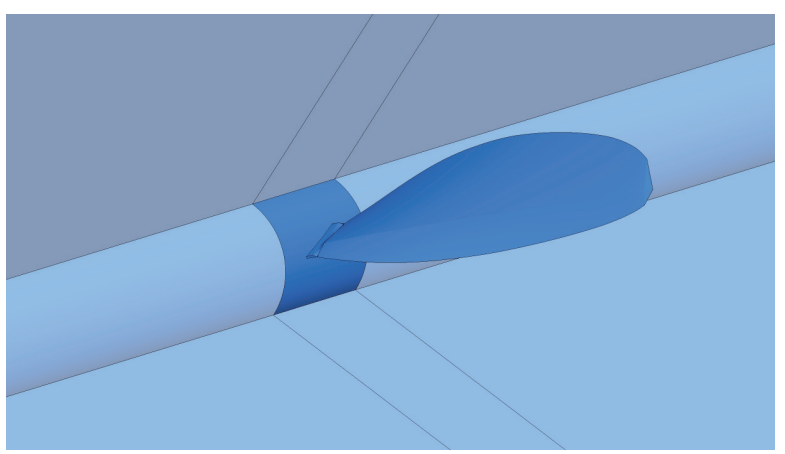

Fig. 3. Computational domain (Magnification around propeller).

other is termed as the skewed blade. The $30 \%$ chord line is normal to the rotating axis for the original blade. Distributions of chord length and twist angle along the blade span are shown in Fig. 1(d). These are defined in $y-z$ plane and these values are common between the original blade and the skewed blade. The shape of the skewed blade was determined as follows: The leading edge of the skewed blade when the variation of $\beta$ from its value at the root is ignored is on a circular arc with the diameter of $0.4 \mathrm{~m}$ as shown in Fig.1 (e). The skewed blade was obtained by twisting the blade shown in Fig.1(e) around $y$ axis by the variation of $\beta$ from its value at the root.

\section{Experimental Method}

Experiments were carried out under various Reynolds numbers of $500<\operatorname{Re}<10000$ using two kinds of wind tunnel. The working fluid of both wind tunnels is air and the effects 

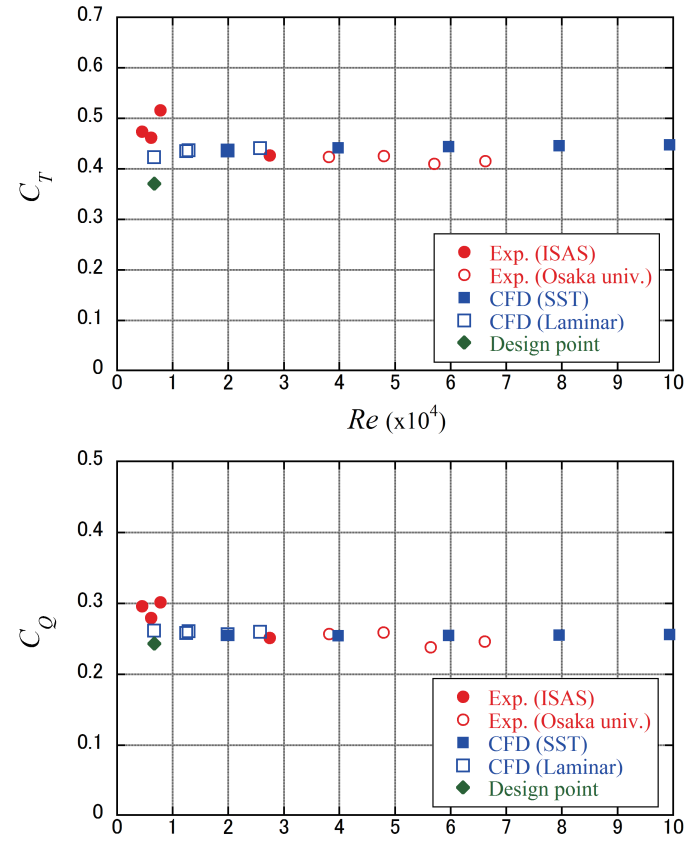

$\operatorname{Re}\left(\times 10^{4}\right)$

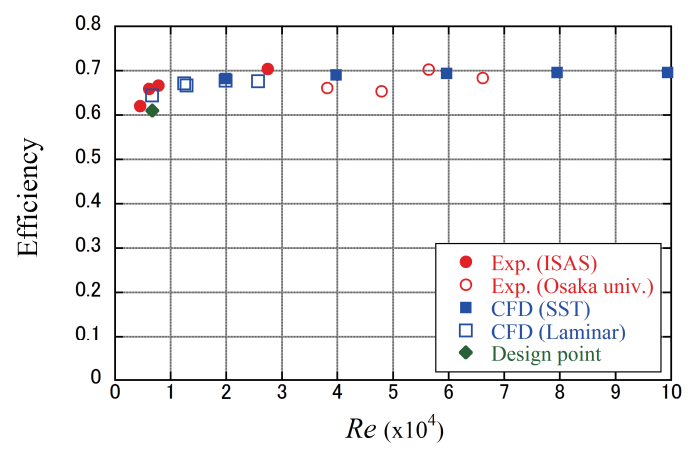

Fig. 4. Propeller thrust coefficient, torque coefficient, and efficiency versus Reynolds number for the original blade.

of Reynolds number are examined. The one is located at Osaka University and the test section of the wind tunnel is a square with width and height of $1.8 \mathrm{~m}$. The wind speed varied between $2 \mathrm{~m} / \mathrm{s}$ and $20 \mathrm{~m} / \mathrm{s}$ and the pressure is kept at atmospheric pressure. The other wind tunnel is located at JAXA-ISAS. In this wind tunnel, the lower Reynolds number flows can be simulated by reducing the pressure.

The thrust and the axis torque of the propellers are measured using two kinds of test stand. The test stand A consists of 5-component force sensor and the propeller thrust is measured. The test stand B consists of a load cell for the thrust measurement and a torque detector. For both test stands, the drag force acting on the propeller support part is withheld from the measured data.

\section{Numerical Method and Validation}

For numerical simulations, ANSYS-CFX 14 is adopted as a flow solver. The flow is assumed to be incompressible. Solver is based on a finite volume method with unstructured mesh and accuracy in space is 2nd order. Shear Stress Transportation (SST) model is adopted as the turbulence model. In addition, calculations where laminar flow is assumed are made. The computational domain is one pitch periodic as shown in Fig. 3 and the length of upstream and downstream the propeller is 2.5 diameter of propeller. Boundary conditions are as follows: The velocity and the static pressure are specified at the inlet and outlet, respectively. The walls are treated as adiabatic and non-slip walls.

Figure 4 shows the thrust coefficient $C_{T}$, the torque coefficient $C_{Q}$, and the efficiency for the original blade obtained from experimental and numerical results. In these figures, the design point means the estimated value by Adkins-Liebeck method. Differences between the two kinds of numerical results are very small. Considerable variation of experimental $C_{T}$ and $C_{Q}$ can be seen at Reynolds numbers of less than 10000. These data were obtained using the wind tunnel at JAXA-ISAS. The small aerodynamic forces due to the low-density working fluid and vibrations of the facility would have reduced the measurement accuracy. However, the experimental and numerical results are in acceptable agreement for discussions. Both experimental and numerical results show that the thrust and the torque coefficients are almost constant in the examined range of Reynolds number and that $C_{T}, C_{Q}$, and efficiencies are larger than the design values.

\section{Flow Patterns and Effects of Backward Sweep Angle of the Blade}

Hereafter, calculation results are shown. In Figs. 5,6,8,9 and 10 , Reynolds number is 20000 , and SST model was used as a turbulence model. And the forward velocity is $2 \mathrm{~m} / \mathrm{s}$ and the working fluid is air at $1 \mathrm{~atm}$ at the far region from the airfoil .

Figure 5 shows relative velocity $[u, v]^{\mathrm{T}}$ fields and propeller-induced velocity $\left[u^{\prime}, v^{\prime}\right]^{T}$ fields with the pressure distribution in the iso-radius planes at $0.5 R$ and $0.8 R$. The relative velocity and the propeller-induced velocity are calculated as follows:

$$
\begin{gathered}
{\left[\begin{array}{l}
u \\
v
\end{array}\right]=\left[\begin{array}{c}
U \\
V-R \omega
\end{array}\right]} \\
{\left[\begin{array}{l}
u^{\prime} \\
v^{\prime}
\end{array}\right]=\left[\begin{array}{c}
U-U_{a} \\
V-R \omega
\end{array}\right]}
\end{gathered}
$$

On the $0.5 R$ plane, a closed recirculation can be seen on the suction surface around the leading edge and the flow attaches on the suction surface as shown in the upper figure of Fig. 5 (a). On the $0.8 R$ plane, a large low-pressure region can be seen around the leading edge on the suction surface although there is no recirculation. In Figs. 5 (b), the propeller induced- fields show distinct leading edge vortex structures at the low-pressure regions. Figure 6 shows the absolute velocity vector field with the pressure distribution on the axial cross section at $25 \%$ chord length. On the suction surface (upper side of the blade cross section), span-wise flows can be seen. The velocity component toward the blade root is large around the blade tip (right side in the figure) and the velocity component toward blade tip is large around the blade root. Consequently, the leading edge vortex occurs with the span-wise flows on the suction surface of the original blade.

Figure 7 shows the thrust coefficient $C_{T}$, the torque 

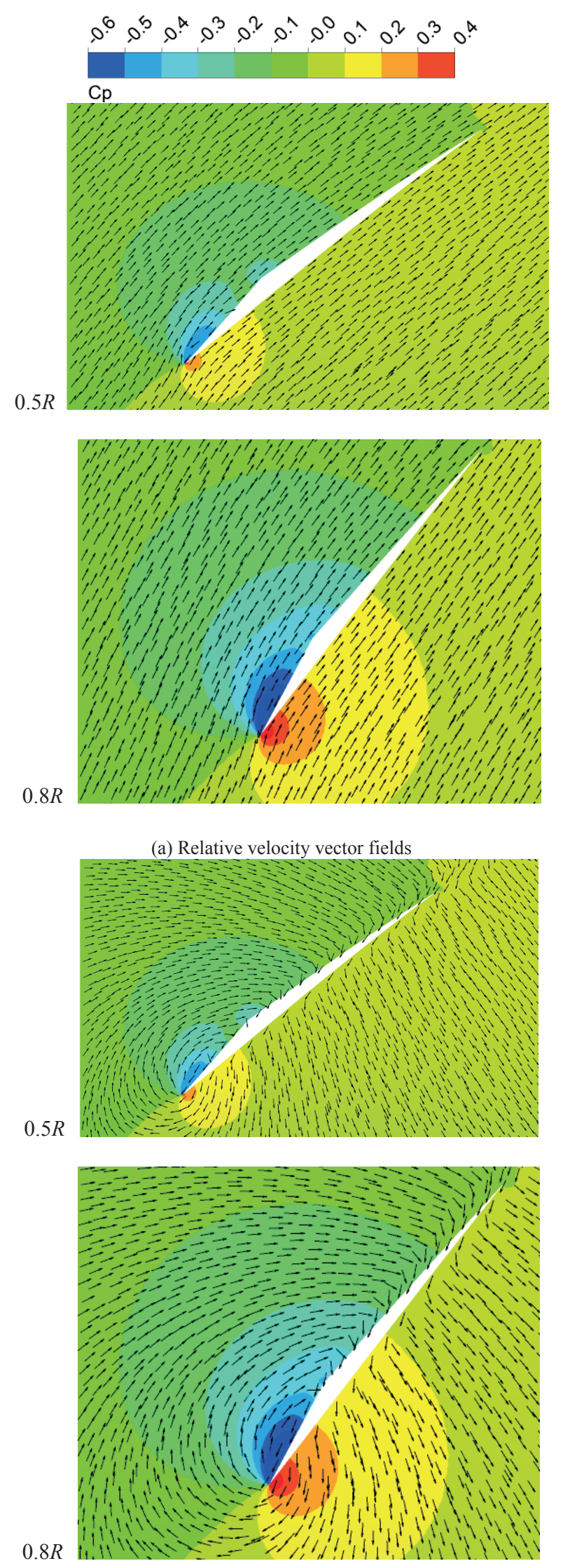

(b) Propeller induced velocity vector fields

Fig. 5. Two kinds of velocity vector field and pressure distribution on iso-radius planes at $0.5 R$ and $0.8 R$.

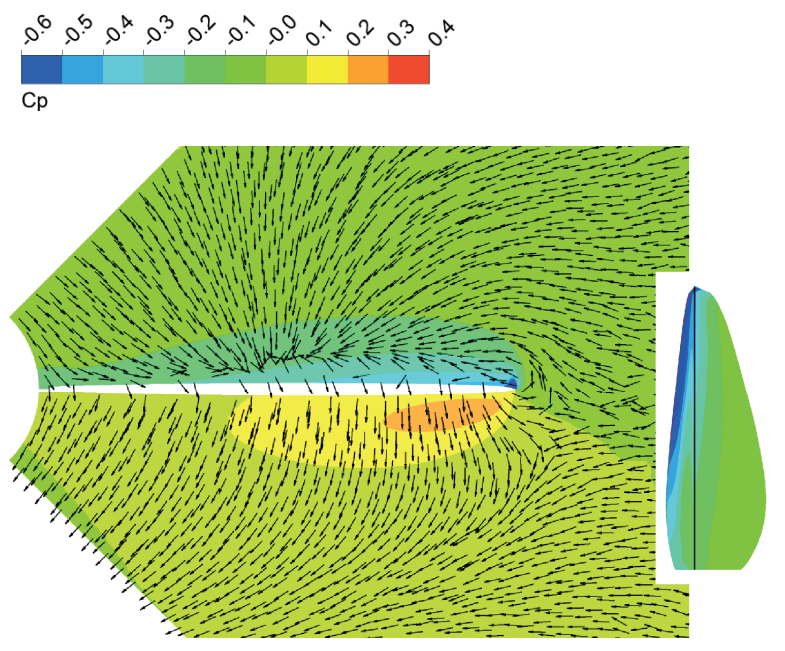

Fig. 6. Absolute velocity vector field and pressure distribution in axial cross section for the original blade.
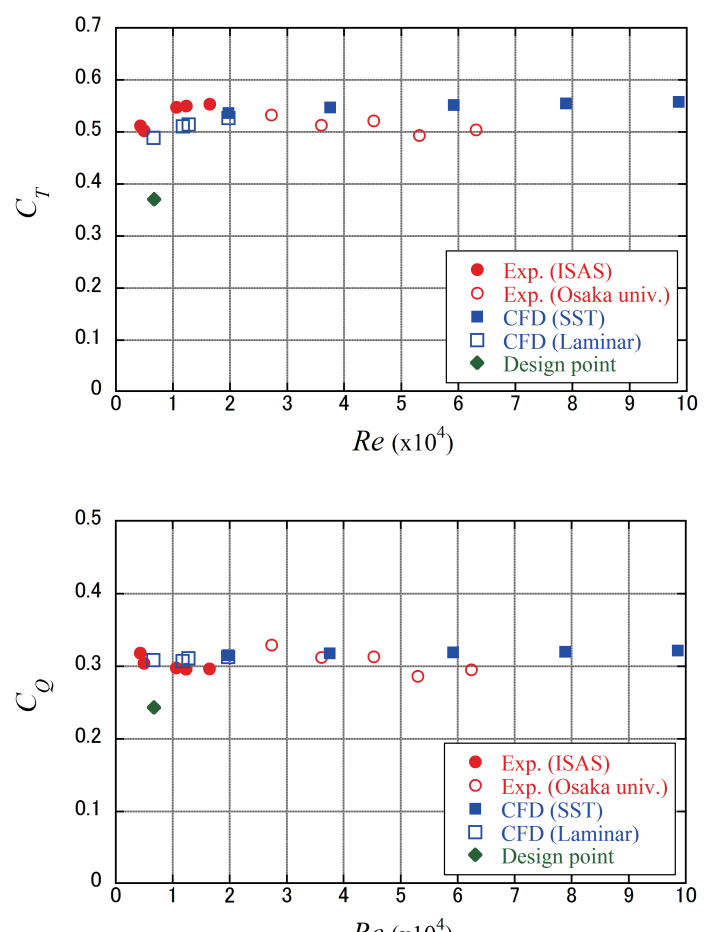

$\operatorname{Re}\left(\times 10^{4}\right)$

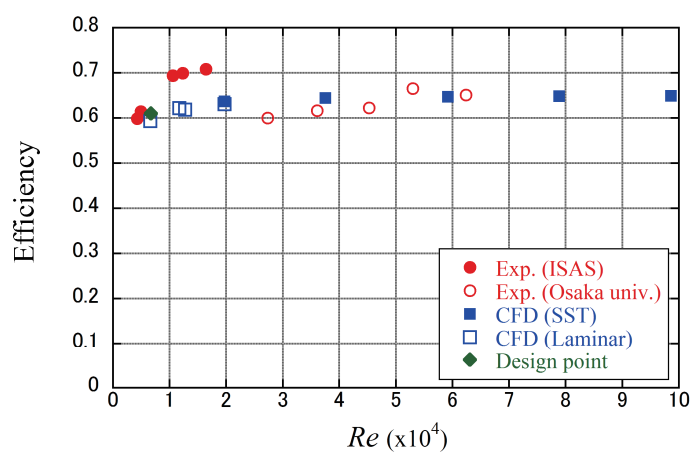

Fig. 7. Propeller thrust coefficient, torque coefficient, and efficiency versus Reynolds number for the skewed blade. 


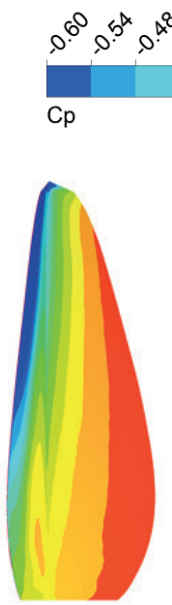

Original blade

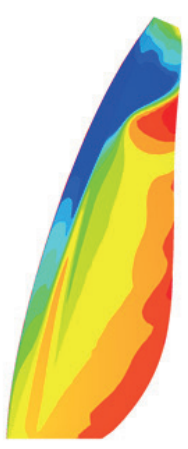

Skewed blade

(a) Suction surface

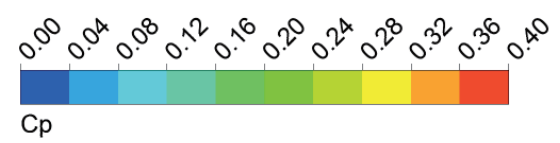

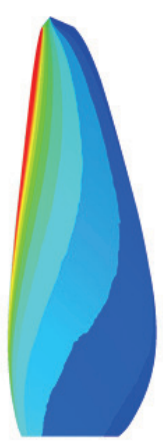

Original blade

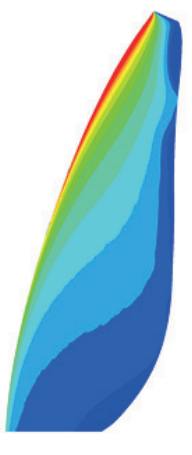

Skewed blade

(b) Pressure surface

Fig. 8. Pressure distribution on blade surface.

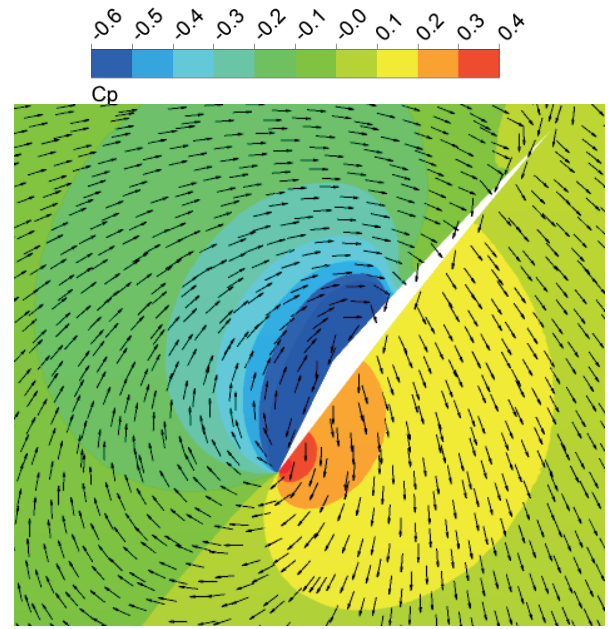

Fig. 9. Propeller-induced velocity vector field and pressure distribution on iso-radius plane at $0.8 R$.

coefficient $C_{Q}$, and the efficiency for the skewed blade obtained from experimental and numerical results. In these figures, the design point means the estimated value by Adkins-Liebeck method without a consideration of the blade

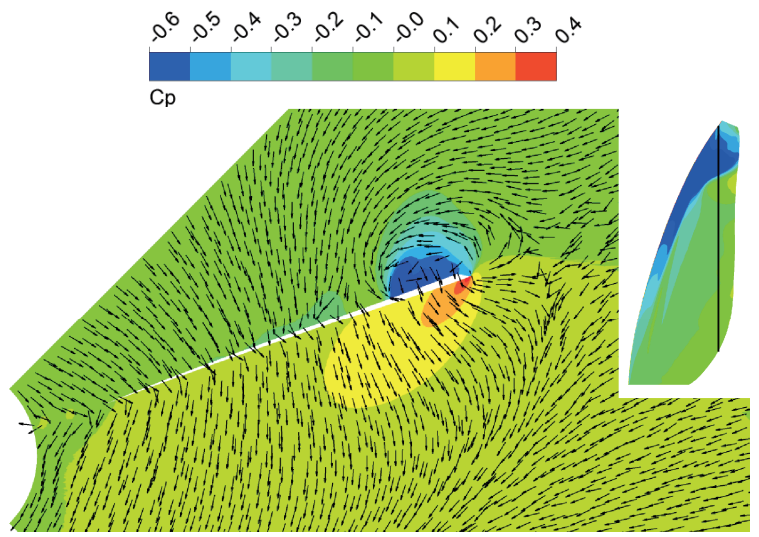

Fig. 10. Absolute velocity vector field and pressure distribution in axial cross section.

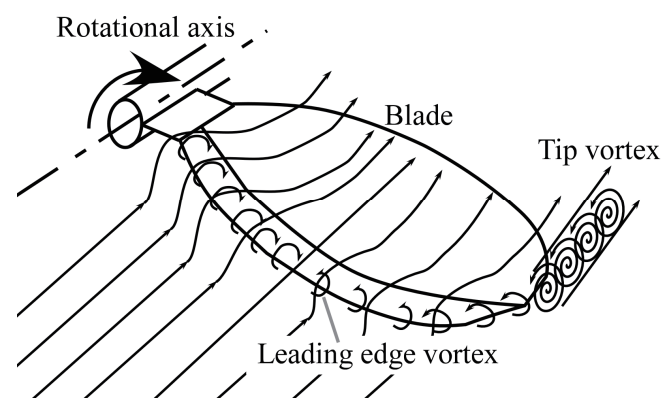

(a) Original blade

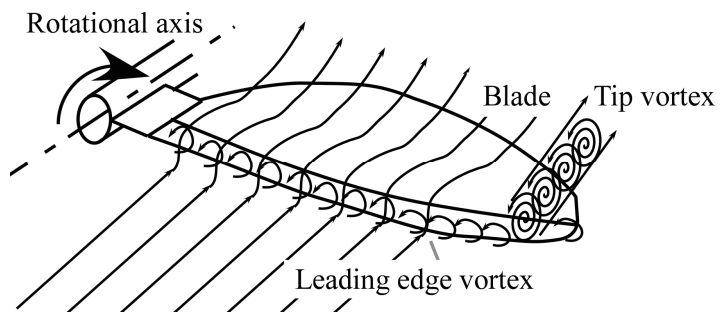

(b) Skewed blade

Fig. 11. Sketches of flow patterns around the propeller blade.

skew effect. These results show that the experimental and the numerical results are in acceptable agreement. Around the design Reynolds number of 6600, the experimental and numerical thrust and torque values are more than $20 \%$ higher than the values of the original blade, which are shown in Fig. 4. However, the propeller efficiency is almost $4 \%$ lower. According to a further investigation ${ }^{8)}$, it has been clarified this $C_{T}$ increase is caused by the difference of the tip speed ratio $\left(=U_{a} / R \omega\right)$ due to the larger radius of the skewed blade than that of the original blade. At the same $\omega$, the tip speed ratio of the skewed blade becomes $6.4 \%$ smaller than that of skewed blade. At the same tip speed ratio, the differences of $C_{T}$ and $C_{Q}$ between the original and the skewed blades are not significant ${ }^{8)}$.

Blade surface pressure distributions are compared in Fig. 8. On the suction surface, large low-pressure region occurs around the blade tip for the skewed blade.

The propeller-induced velocity on the iso-radius plane at 
$0.8 R$ is shown in Fig 9. The large leading edge vortex can be observed. The absolute velocity vector field and the pressure distribution in an axial cross section are shown in Fig. 10, and it can be seen that the large-scale vortex occurs due to the flow from the pressure surface to the suction surface. Consequently, it is found that the large low-pressure region shown in Fig. 9 is caused by the vortex around the blade tip.

The three-dimensional flow patterns around the propeller blade are summarized by sketches in Fig. 11. Around the original blade, the leading edge vortex occurs with inward and outward radial (span-wise) flows along the blade leading edge. The tip vortex occurs outward of the blade tip. Around the skewed blade, the leading edge vortex occurs with the inward radial flow. The tip vortex occurs on the suction surface.

\section{Conclusions}

In the present study, the flow characteristics of the flow patterns around the propeller blade with the low-aspect ratio in low-Reynolds number flows are investigated to develop a high performance propeller of the small scale unmanned aerial vehicle. The results show that the leading edge vortex occurs with span-wise flow on the suction surface of the blade. Consequently the thrust and the torque coefficients and the propeller efficiency become higher than the design values obtained by Adkins-Liebeck method. The influences of the blade planform are examined using the original blade and the skewed blade. The difference of the blade planform compared in the present study does not affect the propeller performance significantly. However, the blade skew affects the tip vortex structures. Around the skewed blade, the tip vortex occurs on the suction surface of the blade and decreases the pressure. Around the blade tip of the skewed blade, the tip vortex yields the thrust force. Such flow patterns were not expected at the design and further investigations are under way.

\section{References}

1) Adkins, C. N. and Liebeck, R. H.: Design of Optimum Propellers, Journal of Propulsion and Power, 10 (1994), pp. 676-672.

2) Azuma, A. and Yasuda K.: Flight Performance of Rotary Seeds, Journal of Theoretical Biology, 138 (1989), pp. 23-53.

3) Lentink, D., Dickson, W. B., van Leeuwen, J. L. and Dickinson, K. H.: Leading-Edge Vortices Elevate Lift of Autorotating Plant Seeds, Science, 324 (2009), pp. 1438-1440.

4) Ellington, C.P. Van den Berg, C., Willmott, A.P. and Thomas, A.L.R.: Leading-edge vortices in insect flight, Nature 384 (1996), pp. 626-630.

5) Dickinson, M.H., Lehmann, F. and Sane, P.S.: Wing rotation and the aerodynamic basis of insect flight. Science 284 (1999), pp. 1954-1999.

6) Srygley, R.B. and Thomas, A.L.R.: Unconventional lift-generating mechanisms in free-flying butterflies. Nature, 420 (2002), pp. 660-664

7) Azuma, A.: The Biokinetics of Flying and Swimming Second edition, AIAA Education Series, AIAA, Reston, Virginia, (2006).

8) Yonezawa, K., Matsumoto, S., Fukumoto, Y., Sunada, S., and Anyoji, M.: Influence of Blade Skew on Propeller Performance in Low-Reynolds Number Flows, Proceedings of AJCPP2014 (2014), AJCPP2014-0105. 